\title{
Preface
}

\section{Molecular Pathology of Bone Tumors: What Have We Learned and How Does It Affect Daily Practice?}

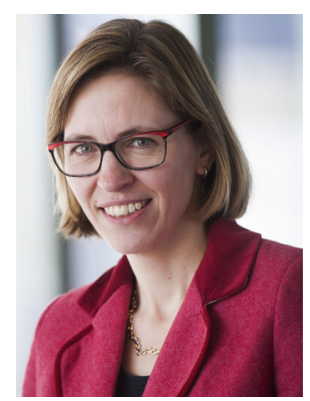

Judith V.M.G. Bovée, MD, PhD Editor

Bone tumors are often found to be difficult by pathologists, because they are rare, and in total, almost 60 entities are recognized with considerable morphologic overlap. Similar to the classification of soft tissue tumors, the World Health Organization 2013 recognizes different categories: benign ( $n=20$ ), intermediate locally aggressive or rarely metastasizing $(n=10)$, and malignant $(n=$ 28), and as such, the different bone tumor entities differ widely in treatment and outcome. In contrast to soft tissue tumors, immunohistochemistry is often of limited value, although in recent years some novel immunohistochemical markers have proven their value. On the other hand, radiologic information is imperative, as for some bone tumors it is not possible to render a definitive diagnosis without taking the exact localization in bone into account. The diagnosis of bone tumors, even more so than in soft tissue, therefore needs to be established in a multidisciplinary team.

Over the past two decades, an increasing amount of genetic data has become available, educating us about the mechanisms involved in the development of bone tumors. For instance, the recent discovery of isocitrate dehydrogenase gene mutations in enchondroma, ${ }^{1,2}$ or histone
H3.3 gene mutations in giant cell tumor of bone and chondroblastoma, ${ }^{3}$ has not only increased our knowledge on the role of epigenetic changes affecting differentiation in the development of bone tumors ${ }^{4}$ but also provided us with novel molecular and surrogate immunohistochemical tools that can help us in the differential diagnosis. 5,6 As such, these molecular data are increasingly important for diagnosis and clinical decision making.

The routine decalcification of bone tumor specimens, which is essential for proper morphology that still remains the cornerstone of the diagnosis, poses a severe limitation when implementing routine molecular testing for bone tumors. Acidbased decalcification methods degrade the nucleic acids and thereby severely compromise molecular testing of bone tumors, causing a very high failure rate. It is therefore imperative that bone tumor labs keep part of the tissue frozen, or use EDTA-based decalcification methods.

This issue of Surgical Pathology Clinics is devoted to bone tumors and addresses diagnostic bone tumor subgroups that often pose difficulties to pathologists (ie, cartilage-forming, bone-forming, giant cell-containing, vascular, 
haematopoietic, and jaw tumors). There is considerable morphologic overlap between the different entities in these subgroups and the use of additional immunohistochemistry, and molecular diagnostics to aid in their distinction are discussed. At the molecular level, recent advances have been made in understanding the molecular background as well as in the identification of molecular targets for therapy in chordoma and Ewing sarcoma. Moreover, the group of rare round cell sarcomas other than classic EWSR1or FUS-rearranged Ewing sarcoma was rapidly delineated with the advent of next-generation sequencing, and their workup using immunohistochemistry and molecular testing in daily practice is discussed. Several reactive and nonneoplastic processes can present primarily in bone in such a way that they pose diagnostic difficulties for the pathologist and can be mistaken for a primary bone neoplasm. In addition, articles are devoted to soft tissue sarcomas that very rarely present as a primary bone tumor, sometimes with specific histologic features (eg, myoepithelial tumors, pseudomyogenic hemangioendothelioma), and, when unaware, the pathologist can make a wrong diagnosis. Finally, with the advent of next-generation sequencing resulting in the detection of somatic but also possible germline variants, and with the recent elucidation that about half of the sarcoma patients have putatively pathogenic monogenic and polygenic variations in known and novel cancer genes in their germline ${ }^{7}$ it is imperative that pathologists are aware of this and that this information is included in multidisciplinary decision making. Thus, this issue of Surgical Pathology Clinics is meant to provide pathologists with state-of-the-art morphologic, immunohistochemical, and molecular tools to increase their diagnostic accuracy of bone tumor diagnosis.

\author{
Judith V.M.G. Bovée, MD, PhD \\ Professor \\ Department of Pathology \\ Leiden University Medical Center \\ PO Box 9600, L1-Q 2300 RC \\ Leiden, The Netherlands
}

E-mail address:

j.v.m.g.bovee@lumc.nl

\section{REFERENCES}

1. Pansuriya TC, van Eijk R, d'Adamo P, et al. Somatic mosaic IDH1 and IDH2 mutations are associated with enchondroma and spindle cell hemangioma in Ollier disease and Maffucci syndrome. Nat Genet 2011;43(12):1256-61.

2. Amary MF, Damato S, Halai D, et al. Ollier disease and Maffucci syndrome are caused by somatic mosaic mutations of IDH1 and IDH2. Nat Genet 2011;43:1262-5.

3. Behjati S, Tarpey PS, Presneau N, et al. Distinct H3F3A and H3F3B driver mutations define chondroblastoma and giant cell tumor of bone. Nat Genet 2013;45(12): 1479-82.

4. Fang D, Gan H, Lee JH, et al. The histone H3.3K36M mutation reprograms the epigenome of chondroblastomas. Science 2016;352(6291):1344-8.

5. Amary MF, Berisha F, Mozela R, et al. The H3F3 K36M mutant antibody is a sensitive and specific marker for the diagnosis of chondroblastoma. Histopathology 2016;69(1):121-7.

6. Luke J, von Baer A, Schreiber J, et al. H3F3A mutation in giant cell tumour of the bone is detected by immunohistochemistry using a monoclonal antibody against the G34W mutated site of the histone H3.3 variant. Histopathology 2017;71(1):125-33.

7. Ballinger ML, Goode DL, Ray-Coquard I, et al. Monogenic and polygenic determinants of sarcoma risk: an international genetic study. Lancet Oncol 2016;17(9): 1261-71. 